\title{
Consolidation in older adults depends upon competition between resting-state networks
}

\author{
Heidi I. L. Jacobs ${ }^{1 *}$, Kim N. H. Dillen ${ }^{1}$, Okka Risius $^{1}$, Yasemin Göreci ${ }^{2}$, Oezguer A. Onur ${ }^{1,2}$, \\ Gereon R. Fink ${ }^{1,2}$ and Juraj Kukolja ${ }^{1,2}$
}

' Cognitive Neuroscience, Research Centre Jülich, Institute of Neuroscience and Medicine (INM3), Jülich, Germany

2 Department of Neurology, University Hospital of Cologne, Cologne, Germany

\section{Edited by:}

Dafin F. Muresanu, University of

Medicine and Pharmacy 'luliu

Hatieganu,' Romania

Reviewed by:

Srikantan S. Nagarajan, University of

California, San Francisco USA

Qiyong Gong, West China Hospital

of Sichuan University, China

\section{*Correspondence:}

Heidi I. L. Jacobs, Cognitive

Neuroscience, Research Centre

Jülich, Institute of Neuroscience and

Medicine (INM3), Leo-Brandt Str. 5,

52425 Jülich, Germany

e-mail: h.jacobs@fz-juelich.de
Memory encoding and retrieval problems are inherent to aging. To date, however, the effect of aging upon the neural correlates of forming memory traces remains poorly understood. Resting-state fMRI connectivity can be used to investigate initial consolidation. We compared within and between network connectivity differences between healthy young and older participants before encoding, after encoding and before retrieval by means of resting-state fMRI. Alterations over time in the between-network connectivity analyses correlated with retrieval performance, whereas within-network connectivity did not: a higher level of negative coupling or competition between the default mode and the executive networks during the after encoding condition was associated with increased retrieval performance in the older adults, but not in the young group. Data suggest that the effective formation of memory traces depends on an age-dependent, dynamic reorganization of the interaction between multiple, large-scale functional networks. Our findings demonstrate that a cross-network based approach can further the understanding of the neural underpinnings of aging-associated memory decline.

Keywords: consolidation, aging, resting-state, memory, networks, fMRI, compensation, competition

\section{INTRODUCTION}

One of the most often expressed complaints of older people is having increasing difficulties with learning and remembering information. Being able to retrieve a specific piece of information from one's memory does not only involve learning of this information, but also consolidating (latin for "to make firm") and storing it. Of all memory systems, episodic memory shows the strongest decline after the age of 60 (Ronnlund et al., 2005). This age-related episodic memory decline has been related to functional changes in distinct brain regions and networks during both encoding and retrieval (Craik and Bialystok, 2006; Daselaar et al., 2006; Miller et al., 2008; Addis et al., 2010; Craik and Rose, 2012). From these studies we know that age-related memory changes are not only associated with local deficits, but more importantly also with changes in large-scale functional brain networks (AndrewsHanna et al., 2007). With aging, these large-scale brain networks show reduced connectivity, efficiency and changes in dynamics such as a reduction in between-network flexibility (Spreng and Schacter, 2012; Yang et al., 2013; Dennis and Thompson, 2014).

Consolidation is the progressive post-acquisition stabilization of long-term memory and the memory phases during which this presumed stabilization takes place (Dudai, 2004). In contrast to encoding and retrieval, consolidation is not associated with a conscious experience or with an explicit cognitive attribute. Therefore, consolidation is difficult to tap into and to date no cognitive paradigm exists that captures the neural correlates of consolidation directly. Traditionally, it is believed that sleep is necessary to consolidate information and to induce changes at the systems level (Euston et al., 2007; Girardeau et al., 2009). Consolidation is thought to involve a repeated spontaneous reactivation associated with the learning event during periods of sleep or rest (McGaugh, 1966). This repeated reactivation stems from the interplay of neural activities between remote brain areas that were already engaged during learning. Reactivation after encoding strengthens memory traces and thereby leads to consolidation (Pennartz et al., 2002).

The standard neurobiological view of consolidation is that the hippocampal system and the relevant neocortical areas are responsible for initial consolidation, but that with time, this information is gradually restructured and becomes integrated in the neocortex and independent of the hippocampus. The hippocampus thus plays a time-limited role. Over time, the hippocampus merely acts as an index, containing only knowledge about which cortical areas are part of that memory, which facilitates retrieval (Squire, 1992; McClelland et al., 1995). An alternative hypothesis, the multiple trace theory, suggests that the hippocampus remains continuously involved in the storage and retrieval of episodic memories (Nadel et al., 2000). In this model, the entire hippocampus-neocortical system encodes the trace in a distributed manner, but with recurrent reactivations, multiple related hippocampal-dependent traces exist. Both hypotheses assume that there is some memory reorganization happening in the brain. However, whether or not the links with 
the hippocampus fade away or are continuously involved differs (Nadel and Bohbot, 2001; Dudai, 2004; Nadel et al., 2012).

One-way to reveal the neural mechanisms underlying consolidation is to investigate blood-oxygenated-level-dependent (BOLD) resting-state functional magnetic resonance imaging (fMRI) connectivity differences over time (Durrant and Lewis, 2009; Vincent, 2009). Several studies have therefore examined change $s$ in the BOLD signal after one or several days (Takashima et al., 2009; Van Kesteren et al., 2010; Vilberg and Davachi, 2013).

Importantly, several studies have investigated the formation of memory traces in response to a motor learning task (Albert et al., 2009; Daselaar et al., 2010) or visuospatial memory tasks (Peigneux et al., 2006; Takashima et al., 2009; Tambini et al., 2010; Van Kesteren et al., 2010; Groen et al., 2011; Vilberg and Davachi, 2013) by comparing the BOLD resting-state signal after and before learning in young individuals. These studies revealed that learning a motor task is associated with increased resting-state connectivity in the frontoparietal and cerebellar networks post-learning (Albert et al., 2009; Daselaar et al., 2010). Learning an episodic memory task is associated with increased hippocampal-neocortical activity after encoding (Peigneux et al., 2006; Tambini et al., 2010; Van Kesteren et al., 2010; Groen et al., 2011; Vilberg and Davachi, 2013). These studies suggest that during consolidation memories become more distributed across brain regions. However, these studies adopted a region-of-interest approach, rather than a network or whole-brain approach. After a longer interval $(24 \mathrm{~h})$, the connectivity between the hippocampus and neocortical areas reduces, but cortico-cortical connectivity increases, providing evidence for the standard neurobiological view of consolidation (Takashima et al., 2009). Investigation of the resting-state BOLD changes immediately after encoding revealed changes in neural activity as well as in connectivity patterns between specific memory-related brain areas, which correlated with memory performance (Takashima et al., 2009; Tambini et al., 2010; Wang et al., 2012). Importantly, these studies reveal that consolidation occurs already soon after the learned event. The timescale of these processes has thus far only been investigated either after encoding or after longer periods (i.e., $24 \mathrm{~h}$ or more).

In summary, to date the time course and spatial extent of these processes remain poorly understood, in particular since most of these studies adopted a region-of-interest analysis rather than a network approach. However, animal studies suggest that consolidation relies on widespread brain networks, distributed topographically in the neocortex (Freeman and Gabriel, 1999). In the current study, we aim to investigate how aging influences the orchestration of different functional networks involved in initial, short-term consolidation. Within initial consolidation we aim to compare age-related temporal changes within a short timescale, minutes instead of hours. As aging is associated with local and global deficits in functional brain networks, we expect consolidation differences involving multiple resting-state networks between younger and older participants early on. This was examined by comparing intrinsic functional connectivity patterns between groups immediately after encoding compared to before encoding. Second, as inter-network flexibility reduces with aging (Spreng and Schacter, 2012), we expected that younger individuals would show higher consolidation-induced-network dynamics than older individuals, i.e., more neocortical involvement in younger than older participants when comparing restingstate activity immediately after encoding with a subsequent time period within the consolidation phase. Finally, to understand the functional relevance of the neural changes during initial consolidation, resting-state connectivity and dynamics were correlated with behavioral retrieval performance.

\section{MATERIALS AND METHODS PARTICIPANTS}

Forty healthy participants were included: 20 older adults (age between 54 and 66 years, 10 women, 10 men) and 20 young participants (age between 23 and 29 years, 10 women, 10 men). Participants were recruited as part of the RIMCAD study (Retroactive Interference during Memory Consolidation in Aging and Dementia) through advertisements. Exclusion criteria were significant medical (e.g., cancer, thyrotoxicosis), neurological (e.g., epilepsy, Parkinson's disease), or psychiatric disorders (e.g., schizophrenia, depression); current use of medication which is known to influence cerebral function (e.g., anti-depressants); pregnancy or any contraindication toward MRI-scanning (e.g., metal implants, claustrophobia). The ethics committee of the Medical Faculty of the University of Cologne approved of the study. Written informed consent was provided by all participants.

All participants underwent an extensive neuropsychological assessment before MRI scanning in order to ensure the absence of cognitive deficits. The assessment included the Mini-mental state examination (MMSE) (Folstein et al., 1975), immediate and delayed recall of the German version of the Verbal Learning and Memory test (VLMT) (Rey, 1694; Helmstaedter and Durwen, 1990), Trail Making Test (TMT) part A and B (Tombaugh, 2004), and Rey Complex Figure Test (CF Rey) (Meyer and Meyers, 1995).

\section{MRI DATA ACQUISITION}

All imaging was performed using a 3.0 Tesla Siemens Trio MRI scanner (Siemens, Erlangen, Germany) equipped with a standard head coil for radio frequency transmission and signal reception. For each participant, we acquired MR images during three resting-state sessions: one session before encoding, one immediately after encoding and one before retrieval (see Figure 1). The resting-state sessions of the after encoding and before retrieval were acquired consecutively without a pause. Each resting-state session consisted of 190 continuous T2*-weighted echo planar imaging $(\mathrm{EPI})$ volumes (repetition time $=2200 \mathrm{~ms}$; echo time $=$ $30 \mathrm{~ms}$; flip angle $=90^{\circ}$; 36 axial slices; matrix $64 \times 64$; voxel size $3.1 \times 3.1 \times 3.0 \mathrm{~mm}$; scan time $=7 \mathrm{~min})$. The second restingstate state session (after encoding) was longer (313 volumes). In order to keep the time windows comparable for the following analyses, we only used the first 190 volumes for the post encoding condition. This resulted in a time gap of $4.5 \mathrm{~min}$ between the after encoding resting-state session and the before retrieval resting-state session (see Figure 1). During the resting-state scan, participants were instructed to keep their eyes closed, to think of nothing in particular, and not to fall asleep. For the taskrelated $\mathrm{fMRI}$, we applied $\mathrm{T} 2{ }^{*}$-weighted echo planar images (EPI) with BOLD contrast, echo time $(\mathrm{TE})=30 \mathrm{~ms}$, repetition time 


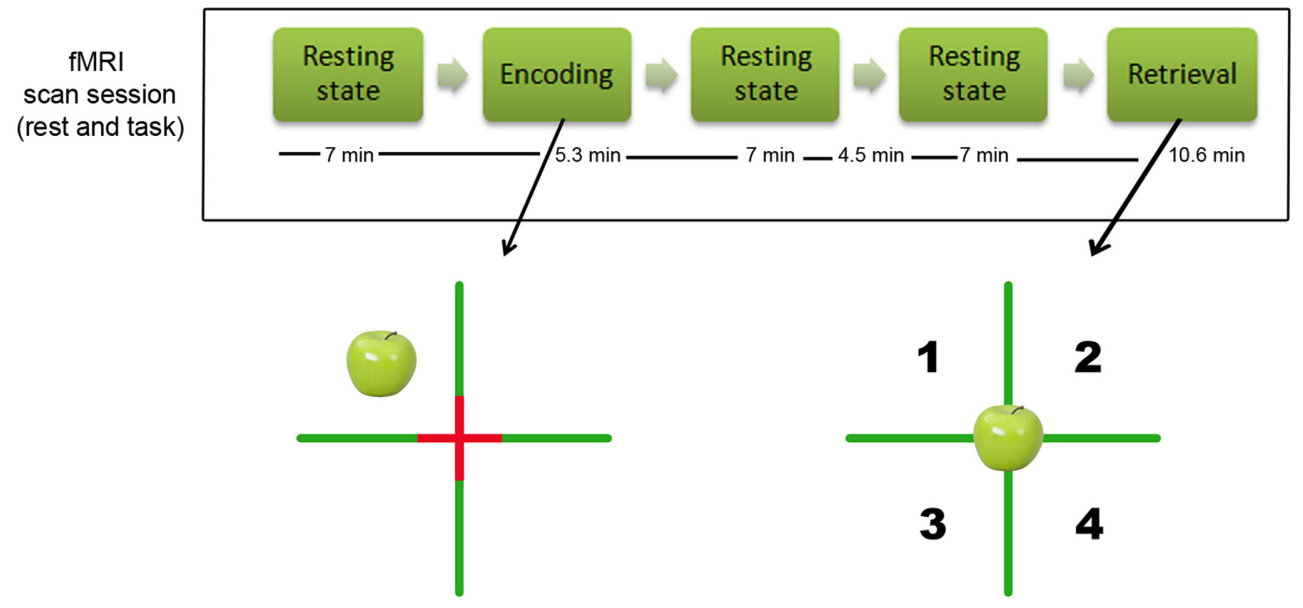

FIGURE 1 | Design of the study. Top of the figure: illustration of the design of the study and timing of the resting-state MR resting state scans. The time indications reflect the duration of each sequence in minutes (note that there was $4.5 \mathrm{~min}$ pause between the two resting-state scans after encoding). Bottom of the figure: illustration of the episodic object-location memory task that participants performed. The left side shows the encoding condition (example of an apple in the left upper corner), the right side shows the retrieval condition in which participants had to indicate whether they had seen the object before, and if so in which quadrant.
$(\mathrm{TR})=2200 \mathrm{~ms}$, flip angle $=90^{\circ}$, slice thickness $3.0 \mathrm{~mm}$, interslice gap $0.3 \mathrm{~mm}$, field of view $(\mathrm{FoV})=200 \mathrm{~mm}$, matrix size $64 \times 64$, inplane resolution $=3.125 \times 3.125 \mathrm{~mm}$. Thirty axial slices per volume covering the brain from vertex to cerebellum were acquired sequentially.

In addition, a high-resolution $\mathrm{T} 1$ anatomical image $(1 \mathrm{~mm}$ isotropic) was obtained for each subject using a threedimensional magnetization-prepared, rapid acquisition gradient echo sequence. The T1-weighted images were used to aid the registration of the functional data into a common standard brain coordinate system (MNI152) and to account for putative atrophy differences between the groups.

\section{EXPERIMENTAL PARADIGM}

We used an adaptation of the spatial Source Memory Task originally employed by Cansino et al. (2002) and previously employed by us (Kukolja et al., 2009). The experiment consisted of an encoding and a retrieval session (see Figure 1). Stimuli consisted of color photographs of common objects. For each subject, a different set of 64 images from a pool of 96 pictures was selected for encoding. Half of the pictures showed artificial (i.e., man-made) objects while the other half presented natural (i.e., not man-made) objects. In the retrieval session, all 96 pictures were presented. This number was used because pilot testing had shown that a large proportion of older participants had difficulties performing the task with more than 100 pictures. During the fMRI encoding session, participants saw a screen which was divided into four quadrants by a green cross with a red fixation cross superimposed at the intersection of the green lines. The 64 objects appeared successively in random order in each quadrant with equal probability (Figure 1). The pictures were shown for $2000 \mathrm{~ms}$, followed by an inter-stimulus interval of $1570 \mathrm{~ms}$ in an event-related design. Participants were asked to memorize all objects and the quadrant in which each object appeared. To ensure proper recognition and visual processing of each object, participants had to indicate with the index or middle finger of their right hand whether the object was "natural," e.g., an animal or a vegetable, or "artificial," e.g., a tool or an instrument. Thirtytwo null events which merely showed the green cross with the red fixation cross superimposed at the intersection were interspersed. This effectively resulted in a variable stimulus-onset asynchrony and allowed a comparison of the BOLD signal of each event type with a no-stimulus baseline (i.e., the null events). The green cross subtended a visual angle of $63.7^{\circ}$ horizontally and vertically. The center of the pictures was located at a visual angle of $\pm 11^{\circ}$ from the horizontal and vertical midline, respectively. The pictures themselves subtended visual angles from $7.3^{\circ}$ to $22.0^{\circ}$ horizontally and from $11.4^{\circ}$ to $19.9^{\circ}$ vertically.

During the fMRI retrieval session, all 96 pictures were presented randomly at the center of the screen (Figure 1). Thus, in addition to the 64 objects previously shown during the encoding session, 32 new objects (16 natural, 16 artificial) were interspersed. The participants' task was to decide whether each picture presented had been shown during the encoding session ("old") or not ("new"). Participants were asked to respond with their left index finger if the picture was new. If the picture was judged as old, participants were asked to indicate the quadrant in which the object had been presented by pressing one of four keys using their right index to little finger. The quadrants were numbered from 1 to 4 ( 1 corresponding to the index finger and 4 corresponding to the little finger) to facilitate response selection. Participants were instructed to make a guess regarding the object's position if they were not sure about the position which the object had occupied during encoding. For subsequent analyses of accuracy and reaction times during retrieval, only items followed by a correct response in the encoding session (i.e., whether the item was artificial or natural) were taken into account. In the retrieval session, the baseline picture also consisted of the green cross with 
the red fixation cross superimposed at its intersection, dividing the screen into four quadrants. Similar to the encoding session, 48 null-events were interspersed randomly. Pictures were presented for $1500 \mathrm{~ms}$, followed by a $2650 \mathrm{~ms}$ inter-stimulus interval in an event-related design. The durations of the encoding and retrieval sessions were 5.3 and $10.6 \mathrm{~min}$, respectively.

Stimuli were shown using Presentation software (Neurobehavioral Systems Inc., San Francisco, CA, USA). Prior to the fMRI experiment, participants performed one training session of the encoding and retrieval tasks outside and another one inside the MR scanner, both with a reduced number of items.

Age-related activation differences during performance of this task have been described in prior work (Kukolja et al., 2009). For the current study, we focused on brain network connectivity during consolidation and its influence on behavioral performance. For reasons of completeness, we have additionally analyzed brain activity during encoding and retrieval parts of the episodic memory task. The methods and results are provided in the Supplemental Data (see Supplemental Methods, Supplemental Data, Supplemental Tables 1, 2 and Supplemental Figures 1-3).

\section{DATA ANALYSIS}

\section{Analysis of behavioral data}

Behavioral data were analyzed with the SPSS version 20.0. Demographic and cognitive group differences were investigated with ANOVA for continuous variables and a $X^{2}$ test for categorical variables. For the functional task, we applied a signal detection analysis to investigate aging related differences in "old" vs. "new" judgments. This analysis determines the sensitivity $d^{\prime}$ representing the ability to classify old items as "old" (Green and Swets, 1966). This distinction cannot be assessed by the comparison of response accuracy. The $d^{\prime}$ index computes the distance between the signal (old as classified as old) and noise (old classified as new) distribution means in standard deviation units. The parameter was calculated as described in (Stanislaw and Todorov, 1999; Kukolja et al., 2009): $d^{\prime}=\Phi^{-1}\left(H^{\prime}\right)-\Phi^{-1}\left(F^{\prime}\right)$ where $H^{\prime}$ is the corrected hit rate, $F^{\prime}$ the corrected false alarm rate, and $\Phi-1$ is the function converting probabilities into $z$ scores. To protect against ceiling effects with $H$ of 1 and $F$ of 0 (corresponding $z$-values would be $+\infty$ or $-\infty$, respectively), we used corrected values of $H$ and $F: H^{\prime}=(h+0.5) /(h+m+1)$ and $F^{\prime}=(f+0.5) /(f+c r+1)$ where $h$ is the number of hits (old as "old"), $m$ the number of misses (old as "new"), $f$ the number of false alarms (new as "old"), and $c r$ is the number of correct rejections (new as "new").

To investigate the functional relevance of the resting-state findings (third hypothesis), partial correlations, corrected for demeaned gray matter volume, between functional connectivity and the experimental task performance ( $d^{\prime}$ prime) were calculated. Statistical significance threshold was set at $p<0.05$.

\section{Data preprocessing}

Data analysis was carried out using FMRIB's Software Library (FSL version 5.0.4; www.fmrib.ox.ac.uk/fsl). Preprocessing consisted of motion correction (MCFLIRT) (Jenkinson et al., 2002), removal of non-brain tissue (using the Brain Extraction Tool) (Smith, 2002), spatial smoothing using a $5 \mathrm{~mm}$ full-width-athalf-maximum Gaussian kernel, and high-pass temporal filtering equivalent to $100 \mathrm{~s}(0.01 \mathrm{~Hz})$. After preprocessing the fMRI volumes were registered to the subject's high-resolution T1-weighted scan using affine registration (FLIRT) with 6 degrees of freedom. Subsequent registration was performed to standard space (MNI152) images using an initial FLIRT affine registration and then further refined with non-linear registration (FNIRT) with a warp resolution of $10 \mathrm{~mm}$ and 12 degrees of freedom. Data were resampled to $3 \mathrm{~mm}^{3}$ resolution.

An overview of all the processing steps of the resting-state data is depicted in Supplemental Figure 4.

\section{Independent component analyses (ICA)}

The concatenated pre-processed data were analyzed using the Multivariate Exploratory Linear Optimized Decomposition into Independent Components (MELODIC) (Beckmann and Smith, 2004) employing the multi-session temporal concatenation ICA to identify large-scale patterns of functional connectivity across participants. This method automatically estimates and decomposes the data from all 120 scans (both groups at three time points) into a set of spatially independent maps each associated with an internally consistent temporal dynamic characterized time course. These spatio-temporal patterns reflect the underlying resting-state networks and artifactual components that give rise to the BOLD signals. The ICA provides intensity values $(z$ scores) and thus a measure of the contribution of the time course of a component to the signal in a given voxel. This allows a voxelwise map of quantitative measures of functional connectivity that can be further statistically analyzed with the dual regression technique. ICA is superior to seed-based correlations for probing large-scale resting-state networks as it is more robust to cardiovascular confounds or motion artifacts (Beckmann and Smith, 2004). The fact that we used the data of all subjects and all time points ensures a robust identification of the components.

We focused on networks relevant to memory or consolidation, viz. the default mode network, the frontoparietal network, the executive network and the salience network. Furthermore, as we used a visuospatial memory task with objects, we also included the ventral stream network and two visual networks. The components of interest were selected based on visual inspection and spatial correlation with a previously defined template of 10 well-matched resting-state components to the BrainMap database (Smith et al., 2009; Laird et al., 2011). To account for noise, components reflecting the white matter and cerebrospinal fluid were included in further analyses (Cole et al., 2010; Birn, 2012). Statistical analyses involving all datasets were carried out using the dual regression procedure.

\section{Dual regression}

To investigate functional connectivity across participants and time points, the individual subject-specific spatial maps and the time courses corresponding to the chosen 10 independent components (IC) (eight components of interest and two components to account for noise) were voxel-wise estimated using the dual regression procedure (Beckmann et al., 2009; Filippini et al., 
2009). First, for each subject, the group-average set of spatial maps was regressed (as spatial regressors in a multiple regression) into the subject's 4D space-time dataset. This resulted in a set of subject-specific time series, one per group-level spatial map (stage 1). Next, those time series were regressed (as temporal regressors, again in a multiple regression) into the same $4 \mathrm{D}$ dataset, resulting in a set of subject-specific spatial maps, one per overall group-level spatial map (stage 2 ). In order to analyze differences between resting-state connectivity before encoding and after encoding, and after encoding and before retrieval, we subtracted individual spatial maps from the stage 2 dual regression. In particular, we created subject-specific maps for "after encoding minus before encoding" and for "before retrieval minus after encoding." First, we analyzed within (young or old) and between (young vs. old) group differences in resting-state connectivity in the 8 components for the time point before encoding, as a reference and to validate our results with the existing literature. For the first hypothesis, we then tested within group (young or old) and between group differences (young vs. old) in resting-state connectivity for the 8 components of interest for the "after encoding minus before encoding" maps with two-sample unpaired $T$-tests. For the second hypothesis, we performed similar analyses, but then for the "before retrieval minus after encoding" maps. As we used the maps of the subtracted time points, these group comparisons reflect the interaction "group by time" (Nieuwenhuis et al., 2011). In all these analyses, gray matter density (see below) was added as a centered covariate. For these analyses, we included all voxels in the brain (whole brain connectivity patterns). To assess within-network functional connectivity differences, we performed the same analyses, but with the spatial maps of the IC as a spatial boundary in the multiple regressions. Non-parametric permutation tests ( 5000 permutations; FSL randomize) were used to detect statistically significant differences within or between the groups (Nichols and Holmes, 2002; Smith et al., 2004). Finally, a family-wise error (FWE) correction for multiple comparisons was performed, implementing threshold-free cluster enhancement (TFCE) (Smith and Nichols, 2009) using a significance threshold of $p<0.05$ (Smith and Nichols, 2009). The regions that showed differences in functional connectivity between groups were visualized by means of FSLview. $z$-Values were extracted from significant clusters of voxels from each individual spatial map (FWE-corrected $p<0.05$ ). These values represent connectivity to the given resting-state network with higher absolute $z$-values reflecting stronger connectivity to the resting-state network. Individual resting-state connectivity values for the clusters of each component were exported to and correlated in SPSS (see Section Analysis of Behavioral Data) with behavioral performance to answer hypothesis 3 . Anatomical labeling was done with the Harvard-Oxford atlas and the Cerebellum atlas available in FSL.

\section{Voxelwise-based gray matter volume correction}

To statistically account for possible effects that could be explained by local structural differences between older and young participants, we added partial volume information of gray matter volume at each voxel as a subject-wise and voxelwise regressor in the dual regression analyses (Oakes et al., 2007). To that end, a four-dimensional (4D) map was created by concatenating every participant (4D) with the feat_gm_prepare script of FSL. The purpose of this method is to isolate the functional changes component which cannot be attributed to anatomical differences and is thus likely due to genuine functional differences.

\section{Between-network connectivity}

Between-network functional connectivity differences were assessed using the FSLNets toolbox (http://fsl.fmrib.ox.ac.uk/ fsl/fslwiki/FSLNets). The following steps were performed. First the respective time courses of all maps in each subject were extracted from the dual regression procedure and normalized by the subjects standard deviations. Time courses of artifactual components (e.g., noise) were regressed out of the individual data. Then, subject-wise correlation matrices of the full and partial correlation of all the remaining resting-state time courses were created. These correlation coefficients were then Fisher $z$-transformed and corrected for temporal autocorrelations. This yields individual correlation matrices of all components, representing neuronal signal corrected for the influences of artifactual components. Partial correlations provide a better approximation for direct connections, while full correlations are more sensitive to both direct and indirect connections (Marrelec et al., 2006; Smith et al., 2011). This is because the partial correlation regresses out the time course of a third region when estimating the correlation between the time courses of two other regions (Smith et al., 2013). Therefore, the partial Fisher z-transformed correlations between networks were extracted for each individual and for the three scanned time points. These were entered into the Statistical Package for the Social Sciences (SPSS Inc., Chicago, IL) version 20.0 for repeated measure ANOVA analyses. The within-group analyses were performed with time as the dependent factor (with three levels). Group-differences were calculated with time as the dependent factor with three levels (before encoding, after encoding and before retrieval) and group as independent factor. Main effect of time and group and the interaction "time by group" were analyzed. This interaction tested a differential change for group differences in network coupling. The assumption of sphericity was checked with the Mauchly test, and, if necessary, a Huynh-Feldt correction was applied. Multiple comparisons were adjusted by applying a Bonferonni correction (FWE-based). Post-hoc repeated contrasts and simple main effects were performed in order to explore the interaction effects.

Individual partial correlation values for the interaction between components were correlated (see Section Analysis of Behavioral Data) with behavioral performance to answer hypothesis 3. Significance threshold was set at $p<0.05$.

\section{RESULTS}

\section{DEMOGRAPHICS AND COGNITIVE PERFORMANCE}

Subject characteristics are shown in Table 1. As to be expected, mean age of the older group differed significantly from that of the young group $(p<0.001)$. Differences in cognitive performance between older and young participants were found for the delayed recognition trial of the VLMT memory test $(p<0.01)$ and the delayed recall of the Rey-Osterrieth Complex Figure Test 
Table 1 | Demographic and cognitive characteristics of the participants.

\begin{tabular}{lcc}
\hline & Older $(\boldsymbol{n}=\mathbf{2 0})$ & Young $(\boldsymbol{n}=\mathbf{2 0})$ \\
\hline Age (in years) & $59.3(6.0)^{* * *}$ & $24.6(2.8)$ \\
Education (in years) & $16.9(3.9)$ & $17.7(2.5)$ \\
Female (in \%) & 50 & 50 \\
MMSE score & $29.4(0.7)$ & $29.6(0.9)$ \\
VLMT learning & $51.1(9.1)$ & $56.4(8.8)$ \\
VLMT memory & & $11.6(3.0)$ \\
$\quad$ delayed recall (number & $10.8(2.8)$ & \\
of words) & & $13.9(1.3)$ \\
$\quad$ delayed recognition & $11.9(3.1)^{* *}$ & \\
(number of words) & & $23.1(7.1)$ \\
TMT-A (s) & $32.3(8.6)$ & $44.1(12.6)$ \\
TMT-B (s) & $64.2(17.9)$ & $35.5(0.8)$ \\
CF Rey score IR & $35.7(1.2)$ & $26.5(4.0)$ \\
CF Rey score DR & $22.1(6.3)^{*}$ & $3.01(0.52)$ \\
Experimental memory & $2.41(0.70)^{* *}$ & \\
task: $d^{\prime}$ prime & & \\
\hline
\end{tabular}

Values are mean (SD). MMSE, Mini-Mental state examination; VLMT, verbal learning and memory test; TMT, trail making test; CF Rey, Rey Complex Figure Test. Differences between both age groups significant at ***p $<0.001, * * p<$ 0.01 and $* p<0.05$.

$(p<0.05)$. Older individuals had a lower $d^{\prime}$ prime $(p<0.01)$ score relative to the young individuals.

\section{IDENTIFICATION OF THE COMPONENTS}

Spatial maps of the components of interest, based on the entire sample, including young and older participants, can be seen in Figure 2. Spatial correlations between our IC components and the Brain Map database (Smith et al., 2009; Laird et al., 2011) were high for the frontoparietal network (right: $r=0.679$; left: $r=$ $0.688)$, the default mode network ( $r=0.834)$, the visual network (medial: $r=0.667$; lateral: $r=0.720$ ), and for the executive network $(r=0.616)$. The ventral and the salience networks were not represented in the 10 well-matched networks and were therefore identified via visual inspection.

\section{WHOLE BRAIN FUNCTIONAL CONNECTIVITY DIFFERENCES}

We first set out to investigate resting-state connectivity between these components and the whole brain during baseline (before encoding) and to compare it with post-encoding consolidation within each group. All reported effects were significant at a voxellevel family-wise error (FWE) adjusted threshold of $p<0.05$, corrected for multiple comparisons and are reported in Montreal Neurological Institute (MNI) coordinates.

Before encoding, the young group and the old group showed widespread high levels of within-group connectivity for all the networks (all $p$ s $<0.001$, see Supplemental Figure 5 and Table 2). For the "after minus before encoding" condition, the young participants showed increased connectivity within the right frontoparietal network $(p=0.009)$ and the default mode network $(p=0.005)$. No significant effects were found within the old group. For the "before retrieval minus after encoding" condition, young and old participants showed no within-group effects (see Figure 3 and Table 2).

We then performed an interaction analysis "group by time point" for the three different time comparisons (see Figure 4). Before encoding, older participants showed higher connectivity between the frontal pole [peak $(x, y, z=15,48,33), p=0.025$ ] and the medial visual network compared to the young group. Furthermore, older participants also showed higher connectivity than younger participants between the left frontoparietal network and regions widespread over the cerebrum and one region in the cerebellum. Largest clusters for this increased connectivity (old $>$ young) were observed in the lateral occipital cortex [peak $(x, y, z=21,-75,39), p=0.013]$, the crus I and II of the cerebellum [peak $(x, y, z=15,-84,-30), p=0.015]$, the anterior cingulate cortex [peak $(x, y, z=18,0,39), p=0.019$ ], fusiform gyrus [peak $(x, y, z=-12,-87,-15), p=0.019$ ], frontal pole [peak $(x, y, z=-36,42,0), p=0.017$ ] and precuneus [peak $(x, y, z=-24,-66,12), p=0.034]$. Before encoding, older participants showed lower connectivity than younger participants between the default mode network and lateral occipital [peak $(x, y, z=30,-57,33), p=0.028$ ], lateral parietal [peak $(x, y, z=-45,-33,30), p=0.029]$, and medial parietal regions [peak $(x, y, z=-15,-51,27), p=0.006$ and peak $(x, y, z=$ $6,-48,48), p=0.024$ ] (see Table 3 and Figure 3). It is important to note that these connectivity patterns replicate previously reported age-related differences in resting-state functional connectivity (Damoiseaux et al., 2008; Sambataro et al., 2012).

In order to answer hypothesis 1 , we examined whether there are age differences in immediate consolidation (after encoding) by focusing on the contrast "after encoding minus before encoding". The dual regression with a FWE correction for multiple comparisons revealed a significant group difference for the salience network with posterior part of the parahippocampal gyrus [peak $(x, y, z=33,-30,-27), p=0.019]$ (see Figure 3).

For the second hypothesis, we investigated the "before retrieval minus after encoding" contrast, and found no significant group differences for any component of interest.

To investigate the functional relevance of the resting-state connectivity group differences found during initial consolidation, we performed correlations between the individually extracted connectivity values and retrieval performance (hypothesis 3 ). Neither in the young, nor in the older group, did we find any significant correlation between the connectivity findings and the performance on the experimental memory task (all $p>0.05$ ).

\section{WITHIN NETWORK CONNECTIVITY DIFFERENCES}

To investigate the robustness of the components involved in consolidation, we examined resting-state connectivity of the same comparisons within the components, FWE-corrected for multiple comparisons.

Before encoding, the young group and the old group showed widespread high levels of within-group connectivity for all the networks (all ps $<0.001$; see Supplemental Figure 6 and Table 4). For the "after minus before encoding" condition, the young participants showed increased connectivity within the default mode network $(p=0.02)$ and the executive network $(p<0.001)$. No significant effects were found within the old group. For the 
Medial visual network
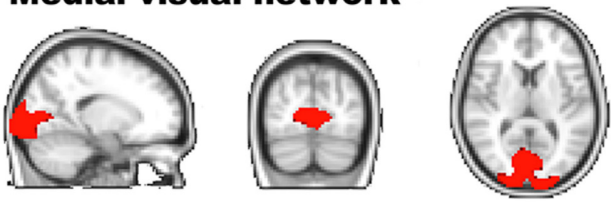

\section{Lateral visual network}
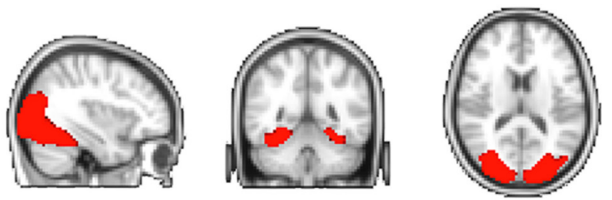

Left frontoparietal network
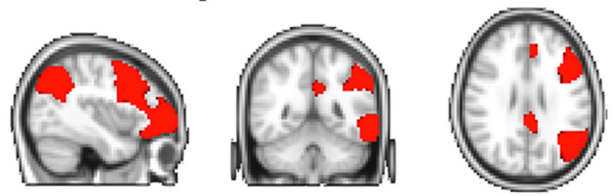

Right frontoparietal network
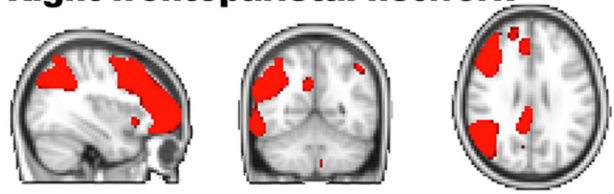

\section{Salience network}
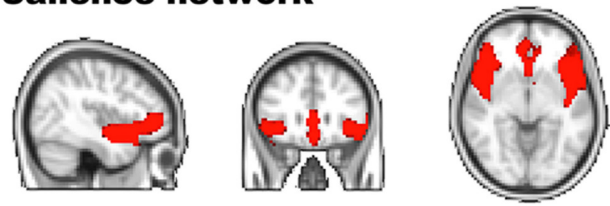

\section{Default mode network}
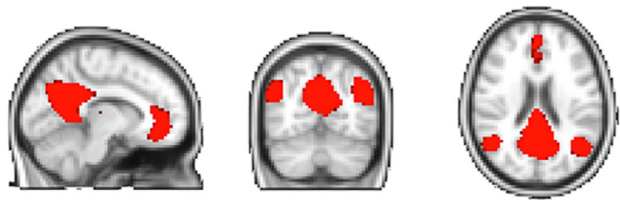

\section{Ventral stream network}
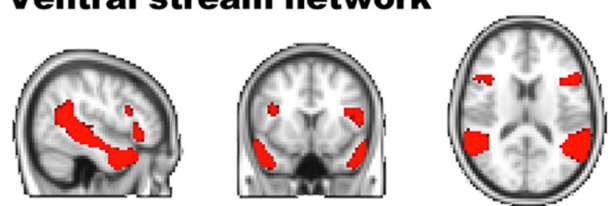

\section{Executive network}
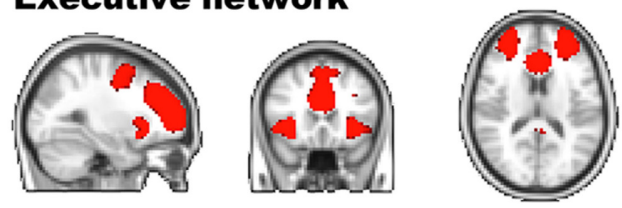

FIGURE 2 | Spatial layout of the independent components of interest of the entire sample. Visualization of the resting-state independent components extracted from the entire sample. For each component, the sagittal, coronal, and transversal perspectives are depicted (radiological convention).

\section{After encoding minus before encoding}

Right frontoparietal network: young
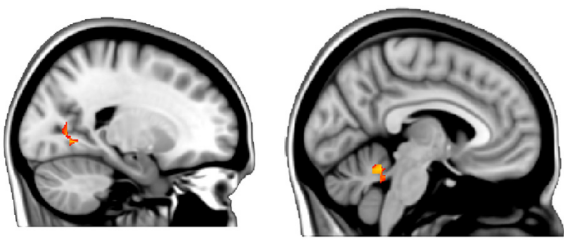

Default mode network: young
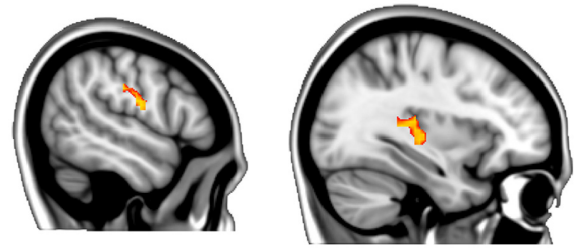

FIGURE 3 | Resting-state differences over time within young participations for the whole brain. Whole-brain within-group effects were found for the contrast "after encoding minus before encoding" in young individuals. Red clusters indicate increased connectivity over time.

"before retrieval minus after encoding" condition, no effects were found within the young or within the old group (see Figure 5 and Table 4).

We then performed an interaction analysis "group by time point" for the three different time comparisons (see Figure 6). For the time point "before encoding," significant group differences were again found in the default mode network and the left frontoparietal network. Older individuals showed less connectivity in the posterior cingulate cortex/precuneus of the default mode network [peak $(x, y, z=-15,-54,27), p=0.003$ ], compared to the younger participants. Older participants showed more connectivity in the frontal pole [peak $(x, y, z=-36,42,0)$, $p=0.005]$ and [peak $(x, y, z=-27,-54,-3), p=0.016]$ and the crus I and II of the cerebellum of the frontoparietal network [peak $(x, y, z=15,-84,30), p=0.012$ ] compared to their younger counterparts.

For the contrast "after encoding minus before encoding," we found no significant group differences within any component (hypothesis 1). For the contrast "before retrieval minus after encoding" (reflecting hypothesis 2), we found less connectivity in the middle temporal gyrus of the ventral stream network [peak $(x, y, z=51,-42,6), p=0.039$ ] for older participants than for younger participants (see Table 5 and Figure 6). No significant correlations were found between the within-network significant connectivity clusters and the retrieval performance on the experimental task (all $p>0.05$; hypothesis 3 ).

\section{BETWEEN NETWORK CONNECTIVITY DIFFERENCES}

The within-group repeated measures analyses for the coupling between the right frontoparietal network and the default mode network revealed for the older individuals $\left[F_{(2,38)}=2.749, p>\right.$ $0.05, \eta=0.126]$ and for the younger participants $\left[F_{(2,38)}=\right.$ 2.505, $p>0.05, \eta=0.117]$ a non-significant effect of time. For 
Table 2 | Resting-state connectivity between networks of interests and the whole brain within the young and older individuals.

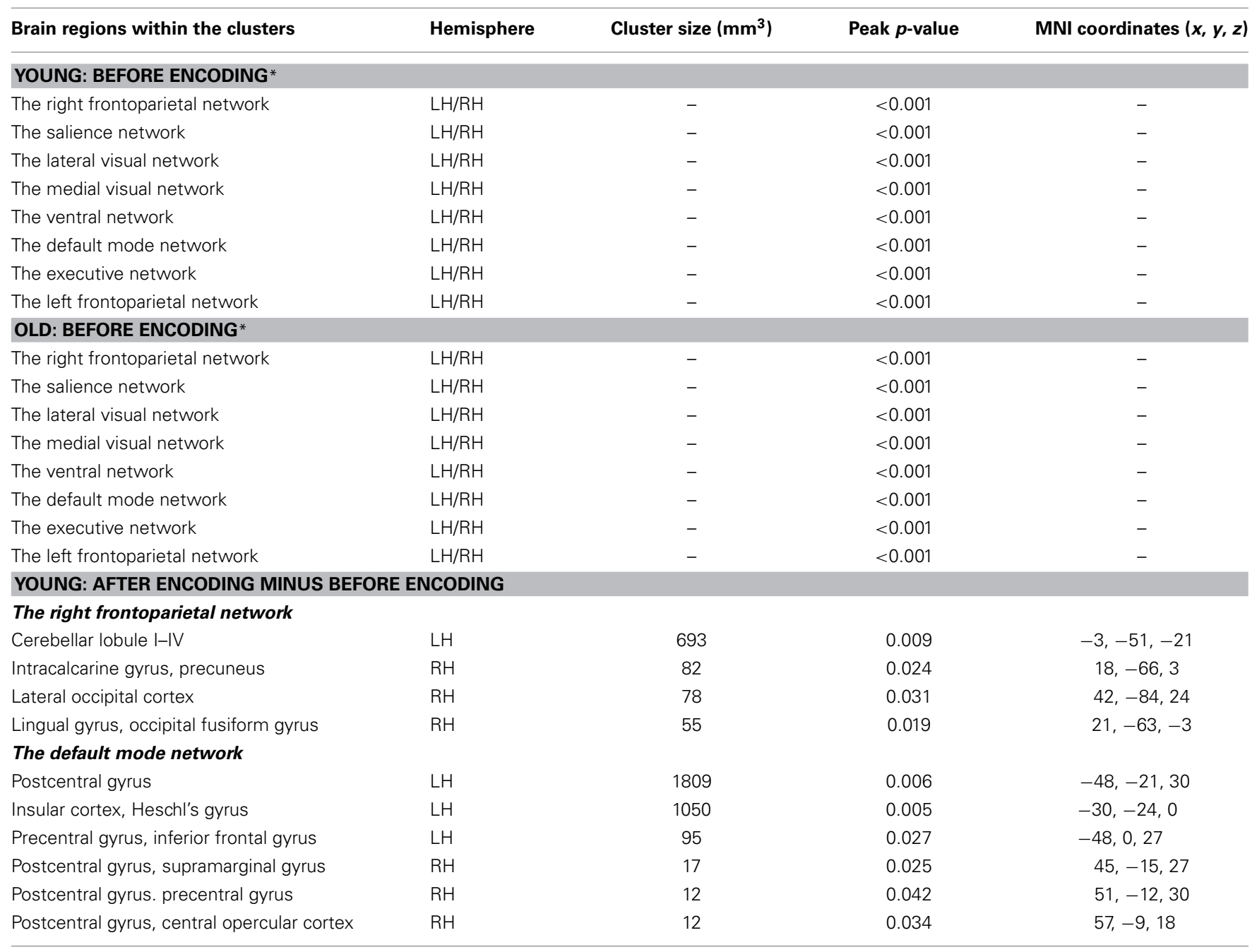

p-Values refer to the peak of all significant voxels. All p-values $<0.05$ are family-wise error (FWE)-corrected at 0.05. Clusters with less than $10 \mathrm{~mm}{ }^{3}$ are not reported. MNI, Montreal Neurological Institute; LH, left hemisphere; RH, right hemisphere. ${ }^{*}$, For the before encoding time, we have not split up the results in clusters, as the effects involved large areas of the total brain and these effects are not the main interest of the manuscript (see Supplemental Figure 5). The Harvard-Oxford (sub)cortical and cerebellum atlas provided within FSL were used as anatomical reference.

the coupling between the executive network and the default mode network, we observed a significant effect of time in the young participants $\left[F_{(2,38)}=8.139, p=0.001, \eta=0.300\right]$; a significant post-hoc contrast was found between "after encoding and before retrieval" $\left[F_{(1,19)}=16.986, p=0.039, \eta=0.205\right]$, but not in the older participants time $\left[F_{(2,38)}=2.694, p>0.05\right.$, $\eta=0.124]$.

Following our second hypothesis, we examined group differences in consolidation over time. The repeated measures ANOVA revealed a significant time by group interaction for the coupling between the right frontoparietal network and the default mode network $\left[F_{(2,76)}=5.179 ; p=0.008, \eta=0.120\right]$, and between the executive network and the default mode network $\left[F_{(2,76)}=\right.$ 9.428, $p<0.001, \eta=0.199$ ] (see Figure 7). As these interactions are disordinal, the main effects should not be interpreted, but are provided for completeness. For the coupling between the right frontoparietal network and the default mode network, the main effect of time was not significant $\left[F_{(2,76)}=0.103 ; p>\right.$ $0.05, \eta=0.003]$ and the main effect of group is also not significant $\left[F_{(1,38)}=0.382, p>0.05, \eta=0.010\right]$. For the coupling between the executive network and the default mode network, we also found no significant main effects for time $\left[F_{(2,76)}=0.517\right.$; $p>0.05, \eta=0.013]$ or group $\left[F_{(1,38)}=0.878 ; p>0.05, \eta=\right.$ $0.023]$. In order to understand the direction of the effects, we have analyzed the simple effects. Post-hoc contrasts (type: repeated) showed that the interaction time by group for the coupling between the right frontoparietal network and the default network was based upon a significant difference between "after encoding" and "before retrieval" $\left[F_{(1,38)}=10.844, p=0.002\right]$. This means that coupling "after encoding" was higher for older individuals than young ones, while the coupling "before retrieval" was higher for the young group compared to the older adults. There was no significant interaction between "before encoding" and "after encoding $\left[F_{(1,38)}=3.60, p>0.05\right]$. Further exploration 


\section{Before encoding}

\section{Medial visual network: old $>$ young}

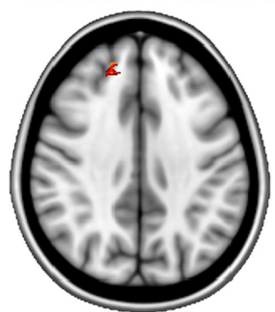

Left frontoparietal network: old > young
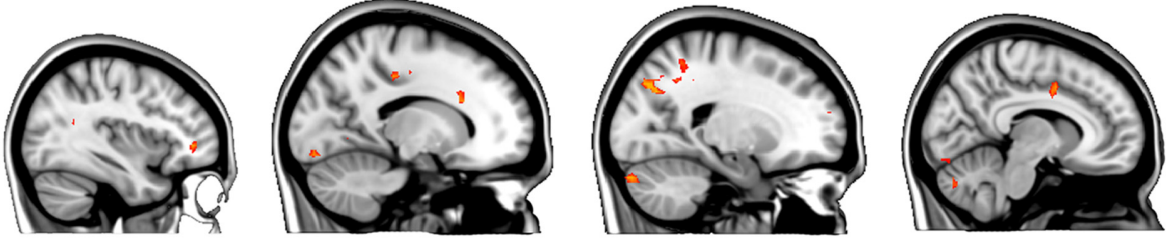

Default mode network: old < young
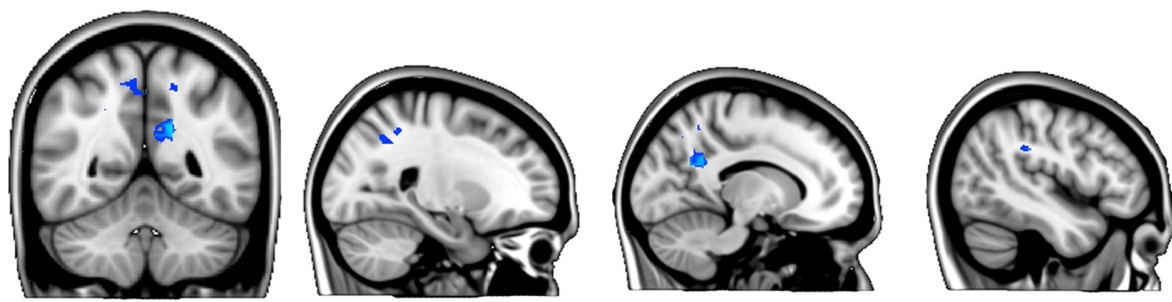

After encoding minus before encoding

\section{Salience network: old > young}
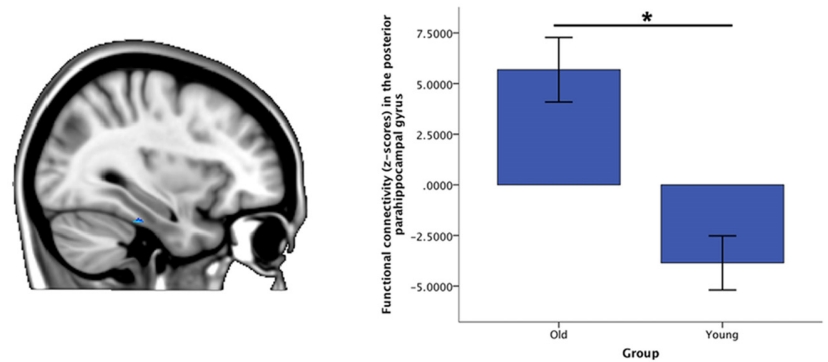

FIGURE 4 | Resting-state differences between older and young participants for the whole brain. Whole-brain group differences were found for the contrasts "before encoding" and "after encoding minus before encoding." Blue clusters show decreased connectivity in older participants, red clusters indicate increased connectivity in older participants. The bar chart depicts the group differences in connectivity between the parahippocampal gyrus and the executive network (error bars indicate 1 standard error). ${ }^{*} p<0.05$. of the simple main effects showed a significant group difference for "before retrieval" (young $>$ old) partial correlations between the networks $\left[F_{(1,38)}=4.498, p=0.041\right]$, but not for "before encoding" $\left[F_{(1,38)}=0.529, p>0.05\right]$ or "after encoding" $\left[F_{(1,38)}=2.390, p>0.05\right]$. There were no within group differences for the paired time point comparisons. For the coupling between the executive and the default mode network, a significant time by group interaction was found between "before encoding" and "after encoding" $\left[F_{(1,38)}=14.987, p<0.001\right]$, and between "after encoding" and "before retrieval" $\left[F_{(1,38)}=\right.$
$16.160, p<0.001]$. Figure 7 shows that there is indeed a higher competition (negative coupling) between these networks "before encoding" for the young group compared to the older group and "after encoding" the competition strength reverses (old > young). "Before retrieval" the competition is higher for the young group than for the older group. Further exploration of the simple main effects showed that the partial correlations between both networks differed significantly between older and young participants for the "before encoding" $\left[F_{(1,38)}=4.306, p=0.045\right]$, "after encoding" $\left[F_{(1,38)}=5.719, p=0.022\right]$, and "before retrieval" 
Table 3 | Group differences in resting-state connectivity of the networks of interest with the whole brain.

\begin{tabular}{|c|c|c|c|c|}
\hline Brain regions within the clusters & Hemisphere & $\begin{array}{l}\text { Cluster size } \\
\left(\mathrm{mm}^{3}\right)\end{array}$ & $\begin{array}{c}\text { Peak } \\
p \text {-value }\end{array}$ & MNI coordinates $(x, y, z)$ \\
\hline \multicolumn{5}{|l|}{ BEFORE ENCODING } \\
\hline Frontal pole, superior frontal gyrus & $\mathrm{RH}$ & 72 & 0.025 & $15,48,33$ \\
\hline \multicolumn{5}{|l|}{ The default mode network (old < young) } \\
\hline Posterior cingulate cortex, precuneus & $\mathrm{LH}$ & 820 & 0.006 & $-15,-51,27$ \\
\hline Precuneus, posterior cingulate cortex, superior parietal lobule & $\mathrm{LH}$ & 90 & 0.029 & $-15,-54,51$ \\
\hline Lateral occipital cortex, angular gyrus & $\mathrm{RH}$ & 141 & 0.028 & $30,-57,33$ \\
\hline Parietal operculum, supramarginal gyrus & $\mathrm{LH}$ & 130 & 0.029 & $-45,-33,30$ \\
\hline Lingual gyrus, occipital pole & $\mathrm{LH}$ & 122 & 0.017 & $-3,-90,-18$ \\
\hline $\begin{array}{l}\text { Angular gyrus, superior parietal lobule, precuneus, lateral occipital } \\
\text { cortex }\end{array}$ & $\mathrm{LH}$ & 38 & 0.035 & $-18,-60,-39$ \\
\hline Anterior cingulate cortex, & $\mathrm{RH}$ & 270 & 0.019 & $18,0,39$ \\
\hline supplementary motor area, & $\mathrm{LH}$ & 76 & 0.023 & $-15,21,24$ \\
\hline paracingulate cortex & $\mathrm{LH}$ & 15 & 0.034 & $-9,36,0$ \\
\hline Occipital fusiform gyrus, lingual gyrus, occipital pole & $\mathrm{LH}$ & 227 & 0.019 & $-12,-87,-15$ \\
\hline \multirow[t]{2}{*}{ Frontal pole } & $\mathrm{LH}$ & 110 & 0.017 & $-36,42,0$ \\
\hline & $\mathrm{LH}$ & 78 & 0.027 & $-27,54,-3$ \\
\hline Precuneus, intracalcarine cortex & $\mathrm{LH}$ & 100 & 0.034 & $-24,-66,12$ \\
\hline Intracalcarine cortex, occipital fusiform gyrus, lingual gyrus & $\mathrm{LH}$ & 95 & 0.029 & $-24,-75,6$ \\
\hline $\begin{array}{l}\text { Posterior cingulate cortex, precentral gyrus, postcentral gyrus, } \\
\text { precuneus }\end{array}$ & $\mathrm{LH}$ & 82 & 0.024 & $-15,-30,42$ \\
\hline Middle frontal gyrus & $\mathrm{RH}$ & 11 & 0.041 & $27,18,39$ \\
\hline
\end{tabular}

p-Values refer to the peak of all significant voxels. All p-values $<0.05$ are family-wise error (FWE)-corrected at 0.05 . Clusters with less than $10 \mathrm{~mm}{ }^{3}$ are not reported. MNI, Montreal Neurological Institute; LH, left hemisphere; RH, right hemisphere. The Harvard-Oxford (sub)cortical and cerebellum atlas provided within FSL were used as anatomical reference.

$\left[F_{(1,38)}=4.679, p=0.037\right]$ time points. Within the young group there were no significant differences in partial correlations between the paired time points. However, the older group showed significant differences between the "before encoding" and "after encoding" ( $p=0.004)$, and the "after encoding" and "before retrieval" ( $p=0.008)$.

To understand the functional relevance (hypothesis 3 ) of these changes in network couplings, we correlated these results with behavioral retrieval scores. Significant correlations between $d^{\prime}$ prime values and the partial correlations between the executive and the default mode network were found for the older group after encoding $(p=0.018)$ and for both groups before retrieval (for old: $p=0.007$; for young: $p=0.002$ ) (see Figure 7).

\section{DISCUSSION}

The aim of this study was to investigate age-related changes during initial consolidation with a focus on large-scale functional networks. We expected age-related differences in initial consolidation by comparing connectivity patterns within relevant resting-state networks after encoding with those before encoding. Second, as consolidation is a dynamic process, we expected agerelated differences over time. To that end, we set out to compare connectivity patterns of resting-state patterns of two different time frames after encoding.

Our results provided evidence that in older participants, compared to younger participants, dynamic changes in functional network coupling occur during the formation of new memory traces, i.e., the initial phase of consolidation. Our findings are consistent with animal lesion studies (Freeman and Gabriel, 1999) which suggested that mnemonic representations change over time and involve multiple functional networks. More importantly, however, our results extend these prior studies by revealing three novel findings: First, while we indeed observed an involvement of multiple, large-scale functional networks, it was the interaction between networks, and not changes within networks, that were related to age-associated differences during consolidation: increased competition between the default mode and executive network and increased cooperation between the default 


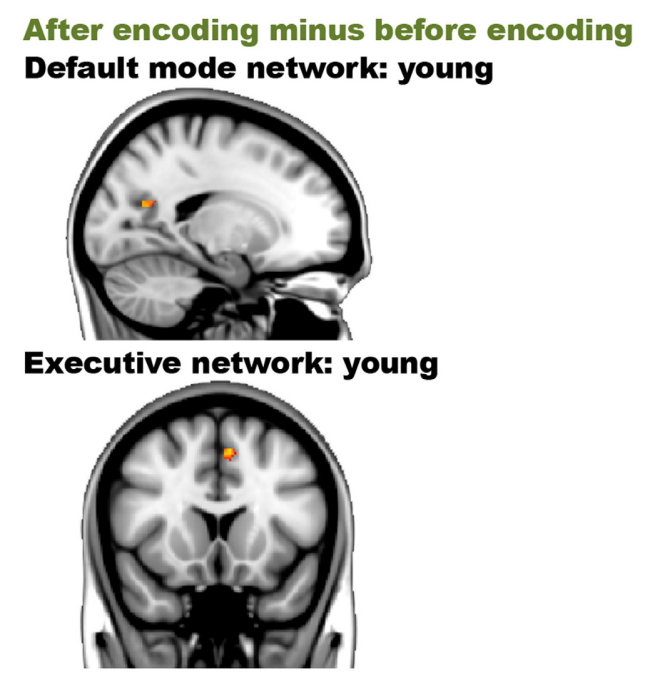

FIGURE 5 | Resting-state differences over time within young participations within the networks of interest. Within-network within-group effects were found for the contrast "after encoding minus before encoding" in young individuals. Red clusters indicate increased connectivity over time. mode and frontoparietal network in the older group compared to the young group were detected during consolidation (Figure 7). Second, this coupling between networks showed a dynamic agerelated reorganization over time, reflected in the non-static level of cooperation or competition between networks over time for both age groups (Figure 6). Third, the magnitude of alterations in coupling between networks was significantly associated with effective consolidation, especially in the older group. Thus, the dynamic organization of resting-state connectivity fluctuations amongst large-scale networks seems to be a powerful tool to measure the formation of new memory traces.

We first set out to investigate age-related differences in initial consolidation across large-scale functional networks. The time frame to stabilize or consolidate memories has always been expected to last weeks to years, but can be separated in an early and long-lasting, also termed synaptic and system consolidation. Synaptic consolidation is a fast process, occurring within the first minutes to hours after encoding. This type of consolidation is assumed to take place in the local nodes of the neuronal circuits that encode the internal representation of the event to be remembered (Dudai, 2004). In contrast, system consolidation, is supposed to take weeks, months or even years before it is achieved. This long-lasting consolidation is thought to induce

Table 4 | Resting-state connectivity within the networks of interests within the young and older individuals.

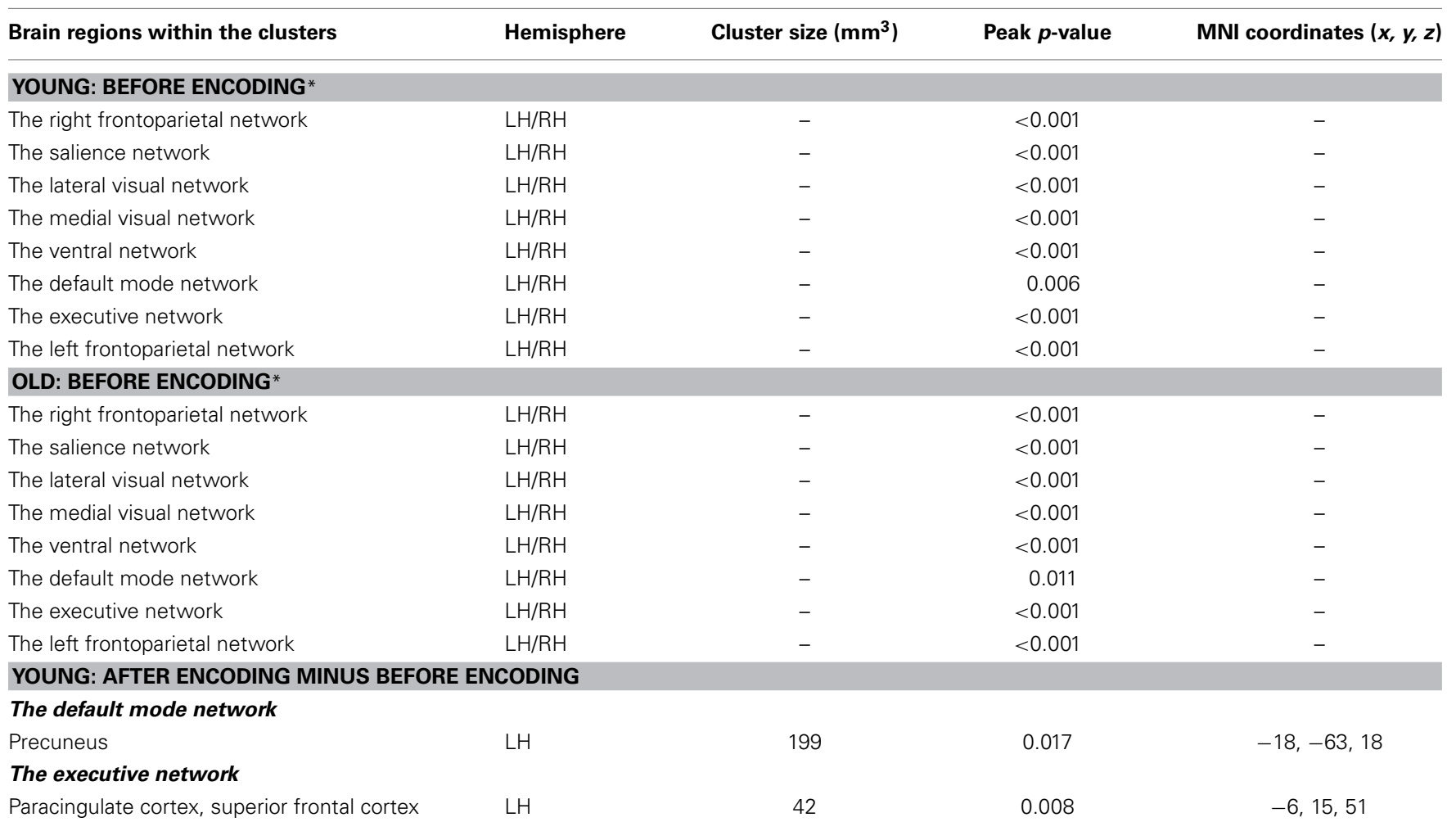

p-Values refer to the peak of all significant voxels. All p-values $<0.05$ are family-wise error (FWE)-corrected at 0.05 . Clusters with less than 10 mm ${ }^{3}$ are not reported. MNI, Montreal Neurological Institute; LH, left hemisphere; RH, right hemisphere. ${ }^{*}$ For the before encoding time, we have not split up the results in clusters, as

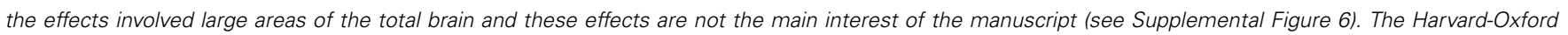
(sub)cortical and cerebellum atlas provided within FSL were used as anatomical reference. 


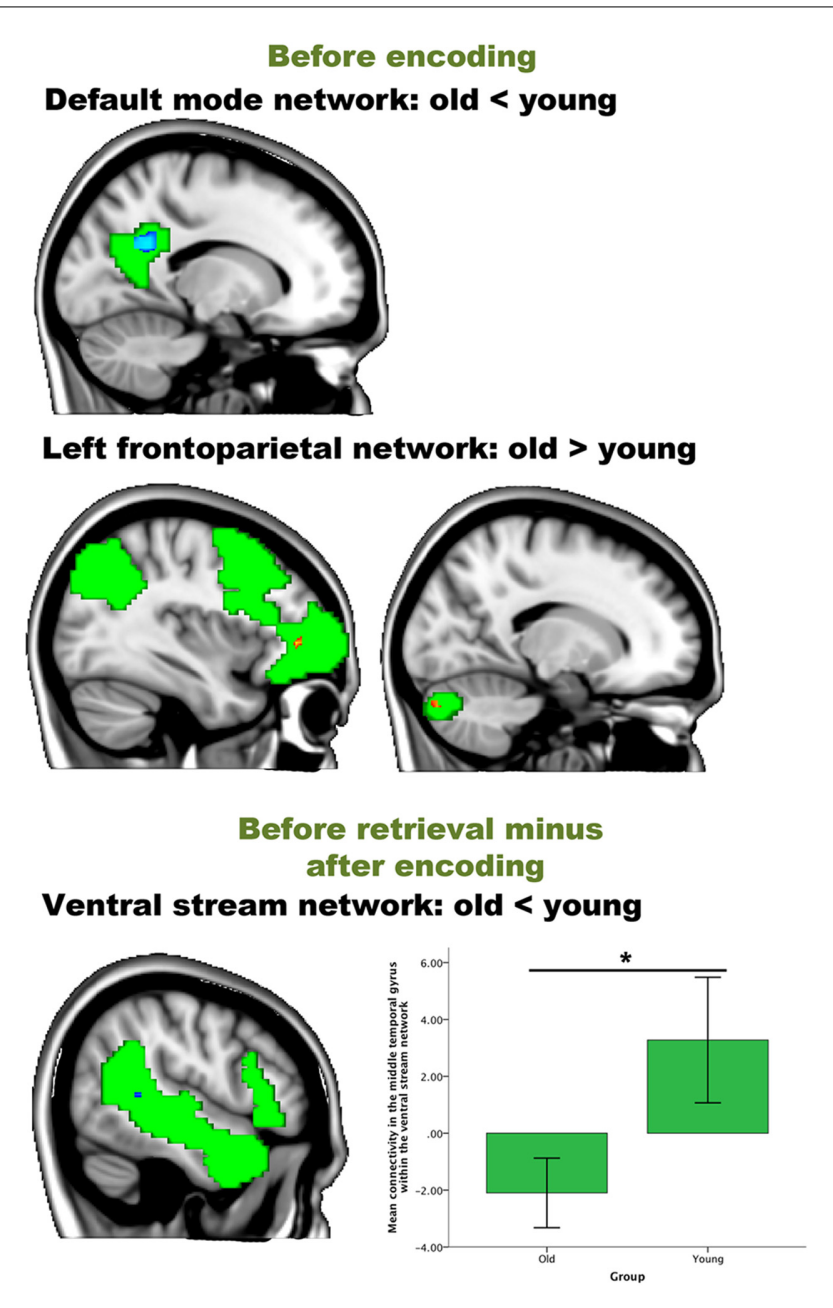

FIGURE 6 | Resting-state differences between older and young within the networks of interest. Within-network group differences were found for the contrasts "before encoding" and "before retrieval minus after encoding." Blue clusters show decreased connectivity in older adults, red clusters indicate increased connectivity in older adults. The bar chart depicts the group differences between the lateral temporal cortex and the ventral stream (error bars indicate 1 standard error) ${ }^{*} p$-value $<0.05$. network reorganization of areas involved in encoding (Dudai, 2004). It has long been thought that sleep is necessary to consolidate information by stimulating specific synaptic connections and removing redundant connections (Euston et al., 2007; Girardeau et al., 2009). Crucially, our results now suggest that new memory traces can indeed be formed in the awake resting-state state (Albert et al., 2009). Furthermore, such network modulations are detectable immediately after presentation of the material to be encoded (Figures 4, 6).

This is, to our knowledge, the first study on age-related neural changes during episodic memory consolidation. Consistent with predictions from Takashima et al. (2009), we found increased connectivity between the parahippocampal gyrus and the salience network in the older compared to the young group (Table 3), showing increased dependence of the medial temporal areas immediately after encoding. The salience network is known to be involved in bottom-up detection of salient events or processes, and mediates the switching between attention and memoryrelated networks (Bressler and Menon, 2010). Older adults seem to rely more on memory-related areas during early consolidation, compared to young adults in the post encoding phase. During the encoding of the items, young adults had higher activity in the parahippocampal gyrus compared to the older individuals (Supplemental Data). This switch in parahippocampal involvement might indicate that neocortical reorganization and independence from the medial temporal lobe areas occurs faster in younger individuals compared to the older group.

The second goal of this study was to investigate the temporal dynamics in age-related differences in consolidation across large-scale functional networks. When investigating changes in intrinsic functional connectivity between before retrieval vs. after encoding, we observed changes in the within-network analyses (Figure 6). Older individuals had lower connectivity values between the middle temporal gyrus and the ventral stream compared to young participants. This might provide additional evidence that consolidation in older individuals at that moment is still hippocampus-dependent. Younger individuals seem to recruit, potentially hippocampus-independent, functionally relevant networks, such as the ventral stream, early on in the consolidation process. The ventral stream is functionally important for form-representation (Kravitz et al., 2011). Our memory task involved memorizing objects and during encoding we did indeed observe activity in the inferior temporal and fusiform gyrus, areas part of the ventral stream (Supplemental Data). Since there was no correlation with retrieval and thus since these neural changes might not necessarily be related to consolidation, we remain cautious with these interpretations.

These findings are on par with prior studies, showing changes in BOLD signal fluctuations during rest indicative of off-line consolidation briefly after learning (Albert et al., 2009). Resting-state states have been shown to be suitable for investigating consolidation (Takashima et al., 2009; Tambini et al., 2010; Wang et al., 2012). However, findings remain controversial with respect to which networks might be involved in memory consolidation. The hippocampus seems to be a central hub for consolidation processes, but both enhanced as well as decreased connectivity to other brain regions has been reported (Takashima et al., 2009; Tambini et al., 2010; Vilberg and Davachi, 2013). Other areas, such as the fusiform gyrus or posterior parietal areas, are most likely related to the task at hand, and might reflect ongoing neocortical integration of the newly learned information. These apparent inconsistencies can be related to differences in analysis approaches (resting-state or task-based, region-of-interest based or network-based), population (only young individuals), or consolidation phase. Whether these neural changes have implications for later memory performance has also led to inconsistencies in the literature. While Tambini et al. (2010), Wang et al. (2012) and Vilberg and Davachi (2013) found correlations with behavioral performance in different brain areas, Takashima et al. (2009) found no correlation.

We found significant correlations with retrieval performance when assessing alterations in the coupling between networks, specifically between the executive and default mode network. 
Table 5 | Group differences in task-free connectivity within the networks of interest.

\begin{tabular}{llccc}
\hline Brain regions within the clusters & Hemisphere & Cluster size $\left(\mathbf{m m}^{\mathbf{3}}\right)$ & Peak $\boldsymbol{p}$-value & MNI coordinates $(\boldsymbol{x}, \boldsymbol{y}, \boldsymbol{z})$ \\
\hline $\begin{array}{l}\text { BEFORE ENCODING } \\
\text { The default mode network (old }<\text { young) }\end{array}$ & LH & 462 & 0.003 \\
$\begin{array}{l}\text { Posterior cingulate cortex, precuneus } \\
\text { The left frontoparietal network (old }>\text { young) }\end{array}$ & RH & 72 & 0.012 & $-15,-54,27$ \\
Crus I and II & LH & 32 & 0.005 & $5,-84,-30$ \\
Frontal pole, orbitofrontal cortex & LH & 12 & 0.016 & $-36,42,0$ \\
Frontal pole & & & $-27,54,-3$
\end{tabular}

The ventral stream network (old before retrieval - old after encoding > young before retrieval - young after encoding)

$\begin{array}{lllll}\text { Middle temporal gyrus, posterior supramarginal gyrus } & \mathrm{RH} & 16 & 0.039\end{array}$

p-Values refer to the peak of all significant voxels. All p-values $<0.05$ are family-wise error (FWE)-corrected at 0.05 . Clusters with less than $10 \mathrm{~mm} \mathrm{~m}^{3}$ are not reported. MNI, Montreal Neurological Institute; LH, left hemisphere; RH, right hemisphere. The Harvard-Oxford (sub)cortical and cerebellum atlas provided within FSL were used as anatomical reference.
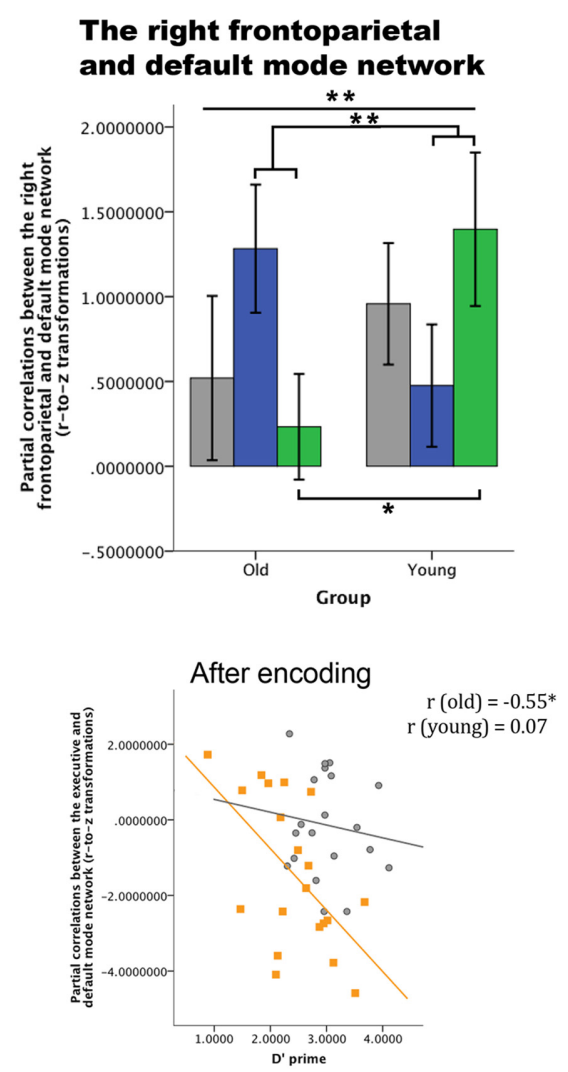

FIGURE 7 | Differences over time between young and old in across-network coupling and the association with memory performance. Top row: partial correlations ( $r$-to-z transformed) between networks over time for both groups (error bars indicate 1 standard error). Bottom row: correlations between on the one hand the partial

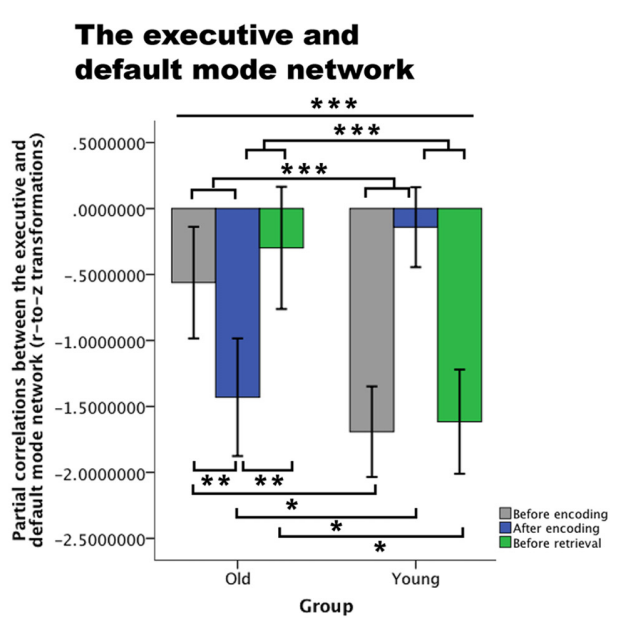

Before retrieval

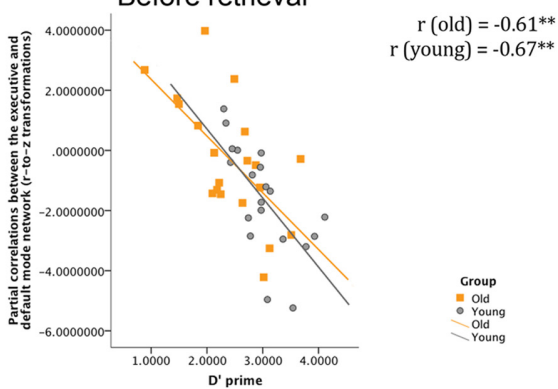

correlations values of the coupling between the default mode network and the executive network, and on the other hand the $d^{\prime}$ prime parameter for both groups. There is a significant association for older adults after encoding and before retrieval, for younger participants only before retrieval. ${ }^{*} p<0.05 ;{ }^{* *} p<0.01 ;{ }^{* *} p<0.001$.
The coupling between the executive and default mode network changed even over a short time period. After encoding, older participants showed increased anti-correlation compared to before encoding and to younger participants. As this correlated positively with retrieval accuracy, the most parsimonious explanation is that the effective formation of memory traces in older participants depends upon suppressing interference from competing networks (Kelly et al., 2008; Wermke et al., 2008). Just 
before retrieval, this competition is reduced, but is still important as indicated by the correlation with retrieval accuracy. This suggests that over a short period of time, first memory traces are laid while being protected from interference from other input. Younger participants are less vulnerable to such interference compared to older participants as indicated by their lower levels of anti-correlation after encoding. There were no significant pairwise changes over time in the young group, suggesting that the level of interference is not influencing consolidation effectiveness in young individuals. However, the significant interaction revealed that young individuals showed more anti-correlation before retrieval compared to after encoding than older individuals. As the before-retrieval network couplings correlated with retrieval performance in young participants, these fluctuations are consistent with existing consolidation theories, suggesting that the executive network becomes gradually independent from the default mode or memory-related network, and that effective memory traces are being formed.

Multiple memory processes cannot be attributed to one or a few brain regions (Buckner and Wheeler, 2001; Craik and Rose, 2012). It is therefore very likely that consolidation depends upon the dynamic interaction between large-scale networks. Our results do not allow a definite conclusion on whether or not these alterations in network coupling reflect a shift from a hippocampus-based network to a neocortical network or that both circuits remain toggling with each other, as long-term consolidation takes days or weeks. Taking these changes into account, it is likely, however, that protein synthesis alterations associated with long-term potentiation occur in this time frame (Reymann and Frey, 2007). Our data contributes to a growing body of literature by showing that consolidation at the system level, i.e., the coupling across networks, occurs already early on and is more strongly related to consolidation than within network changes. Reconsolidation, the susceptibility of memories to change by retrieving or reactivating them, has also been shown to be time dependent and network dependent (Alberini and Ledoux, 2013; Sandrini et al., 2013). However, the focus of this work was to investigate processes during consolidation and not during reconsolidation.

One might argue that participants were actively rehearsing the stimuli for later retrieval and that this effect is reflected in the BOLD signal changes. Actively rehearsing material supports consolidation. We cannot rule this out completely, but consider it unlikely that our participants adopted this strategy as we did not find activation patterns in the encoding phase that can be associated with internal mental repetition (e.g., Broca's areas) (D'esposito et al., 1997; Addis et al., 2007) and we believe that this task consists of too many items to be easily mentally rehearsed. Future studies may take this concern into account this by asking participants about their mental activities or by not informing participants about the goal of the task. Furthermore, we did not include a control session in which participants did neither perform a memory task nor another cognitive task. Importantly, prior work has shown that BOLD fluctuations during rest after learning are a good tool to investigate consolidation (Albert et al., 2009; Vincent, 2009; Daselaar et al., 2010). Another limitation of this study is related to the fact that functional connectivity differences during rest can be related to arousal fluctuations, such as fatigue. Although we did not collect heart rate measurements during scanning, after scanning we asked participants to rate their fatigue levels ( $1=$ no fatigue, $5=$ very fatigued) and whether they believed that they had fallen asleep during scanning. Both groups reported intermediate levels of fatigue [average (standard deviation)] for the three resting-state sessions for the older group: 1.7 (0.7), 2.1 (1.0), 2 (1.2); for the young group: 2.2 (1.2), 3.1 (1.3), 3.1 (1.4). One older subject indicated that she might have slept during the after-encoding and before-retrieval resting-state scan. Thus, even though we cannot rule out completely that fatigue may have influenced our data, its impact is most likely limited.

To conclude, we observed that age-related effective formation of new memory traces does not dependent on specific regions, or specific changes within networks, but rather depends upon the dynamic reorganization of competition and cooperation between resting-state large-scale networks over time. These findings are relevant for our understanding of the neural correlates of agerelated differences in consolidation.

\section{AUTHOR CONTRIBUTIONS}

Heidi I. L. Jacobs, Oezguer A. Onur and Juraj Kukolja were involved in the design of the study. Juraj Kukolja and Yasemin Göreci acquired to the data for this study. Heidi I. L. Jacobs, Kim N. H. Dillen and Okka Risius were responsible for the analyses of this study. Heidi I. L. Jacobs, Juraj Kukolja and Gereon R. Fink interpreted the results. All authors revised the work critically, approved the final version to be published and agreed to be accountable for the accuracy and integrity of the work.

\section{SUPPLEMENTARY MATERIAL}

The Supplementary Material for this article can be found online at: http://www.frontiersin.org/journal/10.3389/fnagi.2014. 00344/abstract

\section{REFERENCES}

Addis, D. R., Leclerc, C. M., Muscatell, K. A., and Kensinger, E. A. (2010) There are age-related changes in neural connectivity during the encoding of positive, but not negative, information. Cortex 46, 425-433. doi: 10.1016/j.cortex.2009.04.011

Addis, D. R., Wong, A. T., and Schacter, D. L. (2007). Remembering the past and imagining the future: common and distinct neural substrates during event construction and elaboration. Neuropsychologia 45, 1363-1377. doi: 10.1016/j.neuropsychologia.2006.10.016

Alberini, C. M., and Ledoux, J. E. (2013). Memory reconsolidation. Curr. Biol. 23, R746-R750. doi: 10.1016/j.cub.2013.06.046

Albert, N. B., Robertson, E. M., and Miall, R. C. (2009). The resting human brain and motor learning. Curr. Biol. 19, 1023-1027. doi: 10.1016/j.cub.2009.04.028

Andrews-Hanna, J. R., Snyder, A. Z., Vincent, J. L., Lustig, C., Head, D., Raichle, M. E., et al. (2007). Disruption of large-scale brain systems in advanced aging. Neuron 56, 924-935. doi: 10.1016/j.neuron.2007.10.038

Beckmann, C. F., Mackay, C. E., Filippini, N., and Smith, S. M. (2009). Group comparison of resting-state fMRI data using multi-subject ICA and dual regression. Neuroimage 47, S148. doi: 10.1016/S1053-8119(09)71511-3

Beckmann, C. F., and Smith, S. M. (2004). Probabilistic independent component analysis for functional magnetic resonance imaging. IEEE Trans. Med. Imaging 23, 137-152. doi: 10.1109/TMI.2003.822821

Birn, R. M. (2012). The role of physiological noise in resting-state functional connectivity. Neuroimage 62, 864-870. doi: 10.1016/j.neuroimage.2012.01.016

Bressler, S. L., and Menon, V. (2010). Large-scale brain networks in cognition: emerging methods and principles. Trends Cogn. Sci. 14, 277-290. doi: 10.1016/j.tics.2010.04.004 
Buckner, R. L., and Wheeler, M. E. (2001). The cognitive neuroscience of remembering. Nat. Rev. Neurosci. 2, 624-634. doi: 10.1038/35090048

Cansino, S., Maquet, P., Dolan, R. J., and Rugg, M. D. (2002). Brain activity underlying encoding and retrieval of source memory. Cereb. Cortex 12, 1048-1056. doi: $10.1093 /$ cercor/12.10.1048

Cole, D. M., Smith, S. M., and Beckmann, C. F. (2010). Advances and pitfalls in the analysis and interpretation of resting-state FMRI data. Front. Syst. Neurosci. 4:8. doi: $10.3389 /$ fnsys.2010.00008

Craik, F. I., and Bialystok, E. (2006). Cognition through the lifespan: mechanisms of change. Trends Cogn. Sci. 10, 131-138. doi: 10.1016/j.tics.2006.01.007

Craik, F. I., and Rose, N. S. (2012). Memory encoding and aging: a neurocognitive perspective. Neurosci. Biobehav. Rev. 36, 1729-1739. doi: 10.1016/j.neubiorev.2011.11.007

Damoiseaux, J. S., Beckmann, C. F., Arigita, E. J., Barkhof, F., Scheltens, P., Stam, C. J., et al. (2008). Reduced resting-state brain activity in the "default network" in normal aging. Cereb. Cortex 18, 1856-1864. doi: 10.1093/cercor/bhm207

Daselaar, S. M., Fleck, M. S., Dobbins, I. G., Madden, D. J., and Cabeza, R. (2006). Effects of healthy aging on hippocampal and rhinal memory functions: an event-related fMRI study. Cereb. Cortex 16, 1771-1782. doi: 10.1093/cercor/bhj112

Daselaar, S. M., Huijbers, W., De Jonge, M., Goltstein, P. M., and Pennartz, C. M. (2010). Experience-dependent alterations in conscious resting state activity following perceptuomotor learning. Neurobiol. Learn. Mem. 93, 422-427. doi: 10.1016/j.nlm.2009.12.009

Dennis, E. L., and Thompson, P. M. (2014). Functional brain connectivity using fMRI in aging and Alzheimer's disease. Neuropsychol. Rev. 24, 49-62. doi: 10.1007/s11065-014-9249-6

D'esposito, M., Detre, J. A., Aguirre, G. K., Stallcup, M., Alsop, D. C., Tippet, L. J., et al. (1997). A functional MRI study of mental image generation. Neuropsychologia 35, 725-730. doi: 10.1016/S0028-3932(96)00121-2

Dudai, Y. (2004). The neurobiology of consolidations, or, how stable is the engram? Annu. Rev. Psychol. 55, 51-86. doi: 10.1146/annurev.psych.55.090902. 142050

Durrant, S., and Lewis, P. A. (2009). Memory consolidation: tracking transfer with functional connectivity. Curr. Biol. 19, R860-R862. doi: 10.1016/j.cub.2009.08.019

Euston, D. R., Tatsuno, M., and McNaughton, B. L. (2007). Fast-forward playback of recent memory sequences in prefrontal cortex during sleep. Science 318, 1147-1150. doi: 10.1126/science.1148979

Filippini, N., Macintosh, B. J., Hough, M. G., Goodwin, G. M., Frisoni, G. B., Smith, S. M., et al. (2009). Distinct patterns of brain activity in young carriers of the APOE-epsilon4 allele. Proc. Natl. Acad. Sci. U.S.A. 106, 7209-7214. doi: 10.1073/pnas.0811879106

Folstein, M. F., Folstein, S. E., and McHugh, P. R. (1975). "Mini-mental state." A practical method for grading the cognitive state of patients for the clinician. $J$. Psychiatr. Res. 12, 189-198. doi: 10.1016/0022-3956(75)90026-6

Freeman, J. H. Jr., and Gabriel, M. (1999). Changes of cingulothalamic topographic excitation patterns and avoidance response incubation over time following initial discriminative conditioning in rabbits. Neurobiol. Learn. Mem. 72, 259-272. doi: 10.1006/nlme.1998.3896

Girardeau, G., Benchenane, K., Wiener, S. I., Buzsaki, G., and Zugaro, M. B. (2009). Selective suppression of hippocampal ripples impairs spatial memory. Nat. Neurosci. 12, 1222-1223. doi: 10.1038/nn.2384

Green, D. M., and Swets, J. A. (1966). Signal Detection Theory and Biophysics. New York: Wiley.

Groen, G., Sokolov, A. N., Jonas, C., Roebling, R., and Spitzer, M. (2011). Increased resting-state perfusion after repeated encoding is related to later retrieval of declarative associative memories. PLoS ONE 6:e19985. doi: 10.1371/journal.pone.0019985

Helmstaedter, C., and Durwen, H. F. (1990). The verbal learning and retention test. A useful and differentiated tool in evaluating verbal memory performance. Schweiz. Arch. Neurol. Psychiatr. 141, 21-30.

Jenkinson, M., Bannister, P., Brady, M., and Smith, S. (2002). Improved optimization for the robust and accurate linear registration and motion correction of brain images. Neuroimage 17, 825-841. doi: 10.1006/nimg.2002.1132

Kelly, A. M., Uddin, L. Q., Biswal, B. B., Castellanos, F. X., and Milham, M. P. (2008). Competition between functional brain networks mediates behavioral variability. Neuroimage 39, 527-537. doi: 10.1016/j.neuroimage.2007. 08.008
Kravitz, D. J., Saleem, K. S., Baker, C. I., and Mishkin, M. (2011). A new neural framework for visuospatial processing. Nat. Rev. Neurosci. 12, 217-230. doi: 10.1038/nrn3008

Kukolja, J., Thiel, C. M., Wilms, M., Mirzazade, S., and Fink, G. R. (2009). Ageingrelated changes of neural activity associated with spatial contextual memory. Neurobiol. Aging 30, 630-645. doi: 10.1016/j.neurobiolaging.2007.08.015

Laird, A. R., Fox, P. M., Eickhoff, S. B., Turner, J. A., Ray, K. L., McKay, D. R., et al. (2011). Behavioral interpretations of intrinsic connectivity networks. J. Cogn. Neurosci. 23, 4022-4037. doi: 10.1162/jocn_a_00077

Marrelec, G., Krainik, A., Duffau, H., Pelegrini-Issac, M., Lehericy, S., Doyon, J., et al. (2006). Partial correlation for functional brain interactivity investigation in functional MRI. Neuroimage 32, 228-237. doi: 10.1016/j.neuroimage.2005.12.057

McClelland, J. L., McNaughton, B. L., and O'reilly, R. C. (1995). Why there are complementary learning systems in the hippocampus and neocortex: insights from the successes and failures of connectionist models of learning and memory. Psychol. Rev. 102, 419-457. doi: 10.1037/0033-295X.102.3.419

McGaugh, J. L. (1966). Time-dependent processes in memory storage. Science 153, 1351-1358. doi: 10.1126/science.153.3742.1351

Meyer, J., and Meyers, K. R. (1995). Rey Complex Figure Test and Recognition Trial. Professional Manual. Lutz, FL: Psychological Assessment Resources Inc.

Miller, S. L., Celone, K., Depeau, K., Diamond, E., Dickerson, B. C., Rentz, D., et al. (2008). Age-related memory impairment associated with loss of parietal deactivation but preserved hippocampal activation. Proc. Natl. Acad. Sci. U.S.A. 105, 2181-2186. doi: 10.1073/pnas.0706818105

Nadel, L., and Bohbot, V. (2001). Consolidation of memory. Hippocampus 11, 56-60. doi: 10.1002/1098-1063(2001)11:1<56::AID-HIPO1020>3.0.CO;2-O

Nadel, L., Hupbach, A., Gomez, R., and Newman-Smith, K. (2012). Memory formation, consolidation and transformation. Neurosci. Biobehav. Rev. 36, 1640-1645. doi: 10.1016/j.neubiorev.2012.03.001

Nadel, L., Samsonovich, A., Ryan, L., and Moscovitch, M. (2000). Multiple trace theory of human memory: computational, neuroimaging, and neuropsychological results. Hippocampus 10, 352-368. doi: 10.1002/1098-1063(2000)10:4<352::AID-HIPO2>3.0.CO;2-D

Nichols, T. E., and Holmes, A. P. (2002). Nonparametric permutation tests for functional neuroimaging: a primer with examples. Hum. Brain Mapp. 15, 1-25. doi: 10.1002/hbm. 1058

Nieuwenhuis, S., Forstmann, B. U., and Wagenmakers, E. J. (2011). Erroneous analyses of interactions in neuroscience: a problem of significance. Nat. Neurosci. 14, 1105-1107. doi: 10.1038/nn.2886

Oakes, T. R., Fox, A. S., Johnstone, T., Chung, M. K., Kalin, N., and Davidson, R. J. (2007). Integrating VBM into the General Linear Model with voxelwise anatomical covariates. Neuroimage 34, 500-508. doi: 10.1016/j.neuroimage.2006. 10.007

Peigneux, P., Orban, P., Balteau, E., Degueldre, C., Luxen, A., Laureys, S., et al. (2006). Offline persistence of memory-related cerebral activity during active wakefulness. PLoS Biol. 4:e100. doi: 10.1371/journal.pbio.0040100

Pennartz, C. M., Uylings, H. B., Barnes, C. A., and McNaughton, B. L. (2002). Memory reactivation and consolidation during sleep: from cellular mechanisms to human performance. Prog. Brain Res. 138, 143-166. doi: 10.1016/S00796123(02)38076-2

Rey, A. (1694). L'examen Clinique en Psychologie. Paris: Presses Universitaires de France.

Reymann, K. G., and Frey, J. U. (2007). The late maintenance of hippocampal LTP: requirements, phases, 'synaptic tagging', 'late-associativity' and implications. Neuropharmacology 52, 24-40. doi: 10.1016/j.neuropharm.2006.07.026

Ronnlund, M., Nyberg, L., Backman, L., and Nilsson, L. G. (2005). Stability, growth, and decline in adult life span development of declarative memory: cross-sectional and longitudinal data from a population-based study. Psychol. Aging 20, 3-18. doi: 10.1037/0882-7974.20.1.3

Sambataro, F., Safrin, M., Lemaitre, H. S., Steele, S. U., Das, S. B., Callicott, J. H., et al. (2012). Normal aging modulates prefrontoparietal networks underlying multiple memory processes. Eur. J. Neurosci. 36, 3559-3567. doi: 10.1111/j.1460-9568.2012.08254.x

Sandrini, M., Censor, N., Mishoe, J., and Cohen, L. G. (2013). Causal role of prefrontal cortex in strengthening of episodic memories through reconsolidation. Curr. Biol. 23, 2181-2184. doi: 10.1016/j.cub.2013.08.045

Smith, S. M. (2002). Fast robust automated brain extraction. Hum. Brain Mapp. 17, 143-155. doi: 10.1002/hbm.10062 
Smith, S. M., Fox, P. T., Miller, K. L., Glahn, D. C., Fox, P. M., Mackay, C. E., et al. (2009). Correspondence of the brain's functional architecture during activation and rest. Proc. Natl. Acad. Sci. U.S.A. 106, 13040-13045. doi: 10.1073/pnas.0905267106

Smith, S. M., Jenkinson, M., Woolrich, M. W., Beckmann, C. F., Behrens, T. E., Johansen-Berg, H., et al. (2004). Advances in functional and structural MR image analysis and implementation as FSL. Neuroimage 23(Suppl. 1), S208-S219. doi: 10.1016/j.neuroimage.2004.07.051

Smith, S. M., Miller, K. L., Salimi-Khorshidi, G., Webster, M., Beckmann, C. F., Nichols, T. E., et al. (2011). Network modelling methods for FMRI. Neuroimage 54, 875-891. doi: 10.1016/j.neuroimage.2010.08.063

Smith, S. M., and Nichols, T. E. (2009). Threshold-free cluster enhancement: addressing problems of smoothing, threshold dependence and localisation in cluster inference. Neuroimage 44, 83-98. doi: 10.1016/j.neuroimage.2008.03.061

Smith, S. M., Vidaurre, D., Beckmann, C. F., Glasser, M. F., Jenkinson, M., Miller, K. L., et al. (2013). Functional connectomics from resting-state fMRI. Trends Cogn. Sci. 17, 666-682. doi: 10.1016/j.tics.2013.09.016

Spreng, R. N., and Schacter, D. L. (2012). Default network modulation and largescale network interactivity in healthy young and old adults. Cereb. Cortex 22, 2610-2621. doi: 10.1093/cercor/bhr339

Squire, L. R. (1992). Memory and the hippocampus: a synthesis from findings with rats, monkeys, and humans. Psychol. Rev. 99, 195-231. doi: 10.1037/0033295X.99.2.195

Stanislaw, H., and Todorov, N. (1999). Calculation of signal detection theory measures. Behav. Res. Methods Instrum. Comput. 31, 137-149. doi: 10.3758/BF03207704

Takashima, A., Nieuwenhuis, I. L., Jensen, O., Talamini, L. M., Rijpkema, M., and Fernandez, G. (2009). Shift from hippocampal to neocortical centered retrieval network with consolidation. J. Neurosci. 29, 10087-10093. doi 10.1523/JNEUROSCI.0799-09.2009

Tambini, A., Ketz, N., and Davachi, L. (2010). Enhanced brain correlations during rest are related to memory for recent experiences. Neuron 65, 280-290. doi: 10.1016/j.neuron.2010.01.001

Tombaugh, T. N. (2004). Trail making test A and B: normative data stratified by age and education. Arch. Clin. Neuropsychol. 19, 203-214. doi: 10.1016/S08876177(03)00039-8

Van Kesteren, M. T., Fernandez, G., Norris, D. G., and Hermans, E. J. (2010). Persistent schema-dependent hippocampal-neocortical connectivity during memory encoding and postencoding rest in humans. Proc. Natl. Acad. Sci. U.S.A. 107, 7550-7555. doi: 10.1073/pnas.0914892107

Vilberg, K. L., and Davachi, L. (2013). Perirhinal-hippocampal connectivity during reactivation is a marker for object-based memory consolidation. Neuron 79, 1232-1242. doi: 10.1016/j.neuron.2013.07.013

Vincent, J. L. (2009). Learning and memory: while you rest, your brain keeps working. Curr. Biol. 19, R484-R486. doi: 10.1016/j.cub.2009.05.02

Wang, D. Y., Liu, D. Q., Li, S. F., and Zang, Y. F. (2012). Increased local synchronization of resting-state fMRI signal after episodic memory encoding reflects off-line memory consolidation. Neuroreport 23, 873-878. doi: 10.1097/WNR.0b013e3283587c96

Wermke, M., Sorg, C., Wohlschlager, A. M., and Drzezga, A. (2008). A new integrative model of cerebral activation, deactivation and default mode function in Alzheimer's disease. Eur. J. Nucl. Med. Mol. Imaging 35(Suppl. 1), S12-S24. doi: 10.1007/s00259-007-0698-5

Yang, A. C., Huang, C. C., Yeh, H. L., Liu, M. E., Hong, C. J., Tu, P. C., et al. (2013). Complexity of spontaneous BOLD activity in default mode network is correlated with cognitive function in normal male elderly: a multiscale entropy analysis. Neurobiol. Aging 34, 428-438. doi: 10.1016/j.neurobiolaging.2012.05.004

Conflict of Interest Statement: The authors declare that the research was conducted in the absence of any commercial or financial relationships that could be construed as a potential conflict of interest.

Received: 13 March 2014; accepted: 15 December 2014; published online: 09 January 2015.

Citation: Jacobs HIL, Dillen KNH, Risius O, Göreci Y, Onur OA, Fink GR and Kukolja $J$ (2015) Consolidation in older adults depends upon competition between resting-state networks. Front. Aging Neurosci. 6:344. doi: 10.3389/fnagi.2014.00344

This article was submitted to the journal Frontiers in Aging Neuroscience.

Copyright (c) 2015 Jacobs, Dillen, Risius, Göreci, Onur, Fink and Kukolja. This is an open-access article distributed under the terms of the Creative Commons Attribution License (CC BY). The use, distribution or reproduction in other forums is permitted, provided the original author(s) or licensor are credited and that the original publication in this journal is cited, in accordance with accepted academic practice. No use, distribution or reproduction is permitted which does not comply with these terms. 